\title{
Hypohidrosis and Dermatological Manifestations as Guides for the Severity of Carpal Tunnel Syndrome
}

\author{
Güldehan Atış ${ }^{1}$, Bit Başak Bilir Kaya ${ }^{2}$ \\ ${ }^{1}$ Department of Dermatology, University of Health Sciences, Haydarpaşa Numune Training and Research Hospital, Istanbul, Turkey \\ ${ }^{2}$ Department of Physical Medicine and Rehabilitation, Government of Health Erenköy Physical Therapy and Rehabilitation Hospital, \\ Istanbul, Turkey
}

\begin{abstract}
Introduction: Carpal tunnel syndrome (CTS) is a common entrapment neuropathy. Hypohidrosis along with some skin findings have been reported to be associated with CTS. Our aims are to detect the incidence of hypohidrosis and accompanying skin manifestations in patients with CTS and to investigate potential relationships between the severity of CTS and the accompanying skin manifestations/hypohidrosis.

Methods: Thirty-four patients with CTS were enrolled in the study. The severity of CTS was divided into three categories: mild, moderate, and severe. The patients were examined to detect skin findings occurring due to CTS. The starch iodine test was applied to measure hypo- or hyperhidrosis.

Results: Of the 34 patients, 29 (85.3\%) had bilateral CTS and 63 hands with CTS were evaluated. Skin manifestations were detected in 36 hands (57.1\%) with CTS. The most common skin findings were contact dermatitis, painful swelling on fingers, and xerosis. Hypohidrosis was detected on 16 hands (47\%) with CTS and was found to be statistically more common on the hands with moderate or severe CTS ( $p=0.000)$.

Discussion and Conclusion: Hypohidrosis is a common finding of CTS and is associated with severe disease. If there is presence of suspicious skin findings and hypohidrosis on hands and fingers, a differential diagnosis of CTS should be kept in mind.

Keywords: Carpal Tunnel syndrome; hypohidrosis; dermatology.
\end{abstract}

C arpal tunnel syndrome (CTS) is a common entrapment neuropathy caused by the compression of the median nerve. Numbness and tingling along the dermatome innervated by the median nerve are common symptoms of CTS ${ }^{[1]}$. Albeit rarer, CTS may also manifest itself dermatologically, in the form of a blistering, painless ulceration on the fingertips, fingertip necrosis, nail discoloration, contact dermatitis, Raynaud's phenomenon, or even autoamputation ${ }^{[2-5]}$. Hypohidrosis is yet another condition reported in conjunction with CTS ${ }^{[6]}$. However, the incidence rates of the accompanying skin findings and hypohidrosis in patients with CTS are still unknown. Our aims in this study are to separately obtain the incidence rates of hypohidrosis and the accompanying skin manifestations in patients suffering from CTS and to investigate potential relationships between the severity of CTS and the accompanying skin manifestations/hypohidrosis. 


\section{Materials and Methods}

Thirty-four patients with CTS were enrolled in the study. The patients were diagnosed with CTS by a specialist in physical therapy and rehabilitation via a clinical examination and electromyographic findings. Approval from a local ethics committee was obtained. Patients who were younger than 18 years of age or who had dermatological or systemic diseases that might lead to hypo- or hyperhidrosis were excluded.

Demographic characteristics such as age, gender, and duration of the disease were recorded. The severity of CTS was divided into three categories: mild, moderate, and severe. The severity of the disease was determined by electromyographic findings. In the first step, the patients' hand(s) (most of the patients had bilateral CTS) were examined by a dermatologist to detect skin findings due to CTS on the first, second, and third fingers. In the second step, the starch iodine test was applied by a dermatologist to measure hypo- or hyperhidrosis on the affected areas and photographs of the affected hands were taken. The first, second, and third fingers were compared with the fourth and fifth fingers. Statistical analyses were performed with SPSS software version 22.0. Mean, standard deviation, minimum, maximum, frequency, and ratio values were used for the determination of data. The Chisquare test, Fisher's exact test, Kolmogorov-Smirnov test and Mann-Whitney $U$ test were used for analyzing the data and a p-value of $<0.05$ was accepted as statistically significant.

\section{Results}

Of the 34 patients, 33 were female (97.1\%) and 1 was male (2.9\%). The mean age of the patients was $55.2 \pm 12.3$ years and the mean duration of disease was $34.9 \pm 35.3$ months. A total of 29 patients (85.3\%) had bilateral CTS (on both hands). As a result, 63 hands with CTS were evaluated in all. Out of these, 28 hands (44.4\%) had mild CTS, 21 hands (33.3\%) had moderate CTS, and 14 hands (22.2\%) had severe CTS. Skin manifestations were detected on 36 hands (57.1\%) with CTS. The observed skin manifestations are summarized in Table 1.

Hypohidrosis was detected on 16 hands (47\%) with CTS. Out of these, 9 hands (56.25\%) had moderate CTS and 7 hands (43.75\%) had severe CTS. Hypohidrosis is statistically more common on the hands with moderate or severe CTS $(p=0.000)$ (Fig. 1). The group with hypohidrosis and the group without hypohidrosis were similar in terms of duration of disease and accompanying skin findings ( $p>0.05$ ) (Table 2 ).
Table 1. Distribution of skin findings on the hands with CTS

\begin{tabular}{lc}
\hline Skin findings & $\mathbf{n}(\%)$ \\
\hline Contact dermatitis & $9(25)$ \\
Painful swelling on fingers & $9(25)$ \\
Xerosis & $4(11.1)$ \\
Longitudinal furrows on the nails & $3(8.3)$ \\
Atrophy & $2(5.5)$ \\
Onychomycosis & $2(5.5)$ \\
Erythema and painful swelling of fingers & $2(5.5)$ \\
Paronychia & $2(5.5)$ \\
Verruca vulgaris & $2(5.5)$ \\
Discoloration of the nail plates & $1(2.8)$ \\
\hline
\end{tabular}

Table 2. Distribution of association of hypohidrosis with the duration of disease, the severity of disease, and accompanying skin findings.

\begin{tabular}{|c|c|c|c|}
\hline & \multicolumn{2}{|c|}{ Hypohidrosis } & \multirow[t]{2}{*}{$\mathbf{p}$} \\
\hline & Absent & Present & \\
\hline Duration of disease (months) & $34.7 \pm 37.9$ & $35.2 \pm 30.7$ & $0.627 \mathrm{~m}$ \\
\hline \multicolumn{4}{|l|}{ Severity of disease (\%) } \\
\hline Mild & $28(59.5)$ & 0 & $0.000 \times 2$ \\
\hline Modarate & $12(25.5)$ & $9(56.25)$ & \\
\hline Severe & $7(14.9)$ & $7(43.75)$ & \\
\hline \multicolumn{4}{|l|}{ Accompanying skin findings (\%) } \\
\hline Present & $27(57.5)$ & $9(56.25)$ & $0.919 \times 2$ \\
\hline Absent & $20(42.5)$ & $7(43.75)$ & \\
\hline
\end{tabular}

m: Mann-Whitney U test; ${ }^{\times 2}$ : Chi-squared test.

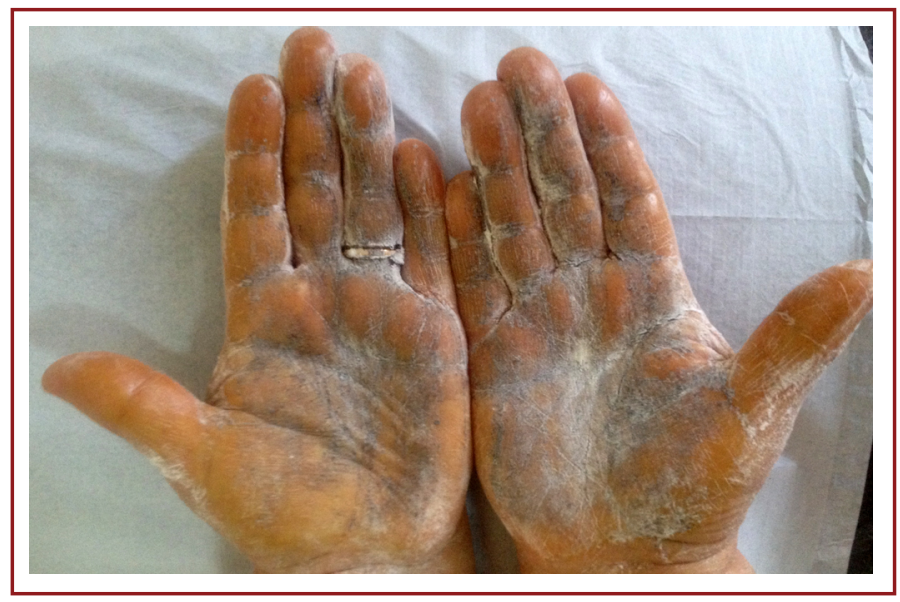

Figure 1. Presence of hypohidrosis on the first and second finger on the left hand with severe CTS and absence of hypohidrosis on the first and second finger on the right hand with mild CTS.

\section{Discussion}

Carpal tunnel syndrome occurs due to compression of the median nerve throughout the carpal tunnel. As a result of this compression, ischemia, mechanical trauma, dysfunctional epidural blood flow, and axonal transport may occur, 
which can consequently induce symptoms such as numbness and tingling sensations along the distribution of the median nerve and weakness and/or atrophy of the musculature. Median nerve compression also leads to autonomic dysfunction. In patients with CTS swelling, an increase in erythema and temperature can occur due to autonomic dysfunction ${ }^{[7]}$. In our study, the most common skin manifestation of CTS was painful swelling of the fingers. Nerve fiber compression can lead to pain along the areas of distribution of the affected nerve. Erythema was detected in only 2 patients with CTS in our study.

Atrophy and xerosis may occur because of digital microcirculatory disorder. Atrophy was detected in 2 patients with CTS in our study ${ }^{[8]}$. Lack of sweating may facilitate xerosis [2]. Xerosis leads to an increased risk of hand eczema and contact sensitization [9]. In addition, peripheral sensitive and autonomic fibers are responsible for the release of neuropeptides such as vasoactive intestinal polypeptides, substance $p$, and calcitonin-related peptide. These peptides play an important role in the pathogenesis of skin inflammation ${ }^{[2]}$ and these pathogenic mechanisms can cause contact dermatitis in patients with CTS. Contact dermatitis was one of the most common skin findings in patients with CTS in our study.

Fingernail changes such as nail plate thickening, dark brown discoloration, transverse furrows, Beau's lines, and cuticle changes have been reported to cause CTS in the literature. In our study, longitudinal furrows on the nails were detected in 3 patients, paronychia in 2 patients, and dark brown discoloration in 1 patient with CTS. Digital microcirculatory defect can cause digital ischemia, which may consequently induce acute periungual inflammation ${ }^{[10]}$ that may be responsible for the nail changes. Sensory dysfunction can lead to mechanic injury, therefore, it may also play a role in the manifestation of nail changes. In our study, verruca vulgaris and onychomycosis were detected in 2 hands. These findings were not thought to be associated with CTS.

Median nerve compression also leads to autonomic dysfunction, which can cause hypohidrosis in the affected areas. Therefore, digital microcirculatory disorder may play a role in the pathogenesis of hypohidrosis in patients with CTS ${ }^{[7]}$. In our study, hypohidrosis was detected in 16 hands (47\%) and was statistically more common on the hands with moderate or severe CTS. Hypohidrosis seems to be associated with the severity of disease in patients with CTS. In conclusion, CTS is one of the most common entrapment neuropathies that is accompanied by skin lesions. Detailed dermatological examination of the hands is important for recognizing the clues of the impending disease. Recognizing specific skin findings due to CTS is important for the dermatologist because early diagnosis can prevent irreversible deformities and bone lesions. Further, it is important for early administration of treatment for the accompanying dermatological disease. Hypohidrosis is a common finding of CTS and it is associated with severe disease. The starch iodine test is an easy and cheap instrument to detect hypohidrosis. In the presence of suspicious skin findings and hypohidrosis on hands and fingers, CTS should be kept in mind by dermatologists as a possible cause.

Our research has some limitations. The study was performed with a limited number of patients, therefore, detailed statistical analysis could not be done for each dermatological finding and the severity of CTS. There was no control group in the study, which would have been helpful in distinguishing non-specific skin lesions from specific skin lesions.

Ethics Committee Approval: Haydarpaşa Numune Training and Research Hospital Ethic Commitee (HNEAH-KAEK 2016/KK/01-03).

Peer-review: Externally peer-reviewed.

Authorship Contributions: Concept: G.A., B.B.K.; Design: G.A.; Data Collection or Processing: G.A., B.B.K.; Analysis or Interpretation: B.B.K.; Literature Search: G.A.; Writing: G.A.

Conflict of Interest: None declared.

Financial Disclosure: The authors declared that this study received no financial support.

\section{References}

1. Miyamoto H, Morizaki Y, Kashiyama T, Tanaka S. Grey-scale sonography and sonoelastography for diagnosing carpal tunnel syndrome. World J Radiol 2016;8:281-7.

2. BaroniA, Piccolo V, Russo T, Ruocco V. Recurrent blistering of the fingertips as a sign of Carpal Tunnel Syndrome: An effect of nerve compression. Arch Dermatol 2012;148:545-6.

3. Ormaechea-Pérez N, Arregui-Murua MA, Zubizarreta-Salvador $J$, Tuneu-Valls A. [Painless ulcers on the fingers: an unusual presentation of severe bilateral carpal tunnel syndrome]. [Article in Spanish] Actas Dermosifiliogr 2012;103:159-60.

4. Fritz TM, Burg G, Böni R. Carpal tunnel syndrome with ulcerous skin lesions. Dermatology 2000;201:165-7.

5. Scarfi F, Arunachalam M, Galeone M, Bassi A, Difonzo E. Fingertip necrosis as a sign of carpal tunnel syndrome. J Am Acad Dermatol 2013;68:51-2.

6. Verghese J, Galanopoulou AS, Herskovitz S. Autonomic dysfunction in idiopathic carpal tunnel syndrome. Muscle Nerve 2000;23:1209-13.

7. Foti C, Romita P, Vestita M. Unusual presentation of carpal tunnel syndrome with cutaneous signs: a case report and 
review of the literature. Immunopharmacol Immunotoxicol 2011;33:751-3.

8. Padua L, Coraci D, Erra C, Pazzaglia C, Paolasso I, Loreti C, et al. Carpal tunnel syndrome: clinical features, diagnosis, and management. Lancet Neurol 2016;15:1273-84.

9. Thyssen JP, Johansen JD, Zachariae C, Menne T, Linneberg A.
Xerosis is associated with atopic dermatitis, hand eczema and contact sensitization independent of filaggrin gene mutations. Acta Derm Venereol 2013;93:406-10.

10. Tosti A, Morelli R, D'Alessandro R, Bassi F. Carpal tunnel syndrome presenting with ischemic skin lesions, acroosteolysis, and nail changes. J Am Acad Dermatol 1993;29:287-90. 